\title{
Bayesian Mindsponge Framework
}

Subjects: Psychology | Social Issues | Others

Contributors: Minh-Hoang Nguyen, QTam-Tri Le

(i)

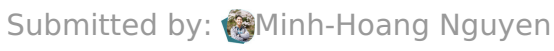

\section{Definition}

Bayesian Mindsponge Framework (BMF, also known as Bayesian Mindsponge analytical approach) is an analytical approach that employs the mindsponge information-processing mechanism and Bayesian analysis (e.g. bayesvl package) as each other's complement to conduct cognitive and psychological research.

\section{Introduction}

A human's brain is a very complex system consisting of approximately 86 billion neurons. Such an enormous nervous system influences and is influenced by other systems of the human body $[\underline{1}]$. To better understand the human's mind, researchers in cognitive and psychological sciences need an adequate analytical approach to scope its dynamic and multiplex mechanism. Recently, Nguyen et al. [2] proposed the Bayesian Mindsponge Framework as a potential approach.

The Bayesian Mindsponge Framework (BMF, also known as Bayesian Mindsponge analytical approach) is an analytical approach that employs the mindsponge information-processing mechanism [3]][4] and Bayesian analysis (e.g. bayesvl package) ${ }^{[5]}[\underline{6}]$ as each other's complement to conduct cognitive or psychological research. In other words, the approach is a combination of the mindsponge mechanism's ability to explain the dynamics and multiplexity of an individual's thoughts and behaviors and the Bayesian analysis method (aided by the Markov Chain Monte Carlo (MCMC) algorithms)'s extreme flexibility in constructing and fitting the model.

BMF was first used to offer a new information-processing approach to study the formulation mechanism of suicidal ideation in an individual's mind [2]. A psycho-religious mechanism was later proposed by Vuong, Nguyen, and Le $[\underline{7}]$ to explain the psychology of suicide attackers using a trust-based evaluation mechanism and cost-benefit judgment among religious individuals. Besides, the information-processing approach was also expanded to the disciplines of educational psychology by exploring the pathways to book reading interest among secondary school students []] and environmental psychology by

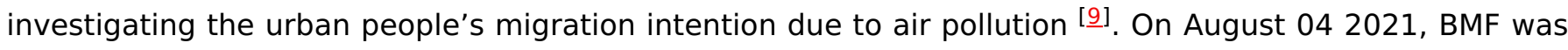
presented at the Applied Statistics Seminar, organized by the Vietnam Institute for Advanced Study in Mathematics (VIASM) [1ㅇ․

\section{Bayesianism and Mindsponge: "a couple"}

The Bayesian inference and Mindsponge notions are a good fit for each other for several reasons. Regarding the Mindsponge mechanism []][]ㅡ, three prominent characteristics can be listed as follows:

1. The Mindsponge process is influenced by both external information and internal preferences (or the mindset's cost-benefit judgment).

2. The causal nexus within the Mindsponge is non-linear rather than linear due to the multiplex nature of information processing.

3. The Mindsponge process is updating in nature through inductive reasoning.

Because of these characteristics, not many statistics approaches can effectively investigate Mindsponge phenomena following its principles. Among the applicable methods, Bayesian inference stands out as an enabler of the philosophy. Firstly, the Bayesian computation aided by the Markov Chain Monte Carlo (MCMC) algorithms offers researchers extreme flexibility in constructing the model. 
Specifically, the MCMC algorithms iteratively generate a large number of samples that help estimate the posterior distribution of any parameters with high precision [11][12]. This advantage enables a wide range of models that can be fitted, such as multilevel models, non-linear model frameworks, or a mixture of both. Therefore, it allows researchers to take the first and second characteristics of Mindsponge philosophy into consideration.

Secondly, Bayesian inference treats all properties as probability $[\underline{13}]$, which permits researchers to look solely at certain aspects of a whole picture without controlling other parameters. Bayesian inference can help the researchers follow the parsimony principle (or Occam's razor), which advocates "entities should not be multiplied beyond necessity" [1ㄴ] . Given the dynamics, multiplexity, and updating manner of the Mindsponge process, the number of control parameters needed for reducing the model's residual might be an enormous number that substantially increases the research cost and eventually reduces the research feasibility $[\underline{15}]$.

Moreover, the higher number of control variables are added, the easier scientific misconduct can be actualized. For example, inappropriate practices, such as stargazing, p-hacking, and HARKing, can be conducted by capitalizing the sample-to-sample variability in the $p$-value [16][1]][18]. Hence, besides assisting researchers in framing research matters, Bayesian inference can also help reduce the persistent reproducibility crisis in psychological research [미][으.

Thirdly, the Bayesian belief updating process plays a fundamental role in following the Mindsponge philosophy. Humans live in a world with an excessive amount of information being continuously updated, so their psychological traits and behaviors also change rapidly depending on the context and personal demand (or mindset's preferences).

As a result, there are possibilities that published scientific findings can be outdated and cannot be replicated after some time. With the belief updating function, Bayesian inference is, thus, a well-fitted methodology for studying cognitive and psychological matters using the Mindsponge philosophy.

\section{Steps to perform the BMF}

The BMF has five steps. However, before these steps, the user has to understand the Mindsponge mechanism's components and structure. Such information can be found here [ㅍ][]].

\section{Step 1 (Identifying research problem)}

Firstly, the user needs to determine the subject or problem they want to investigate. As the information exchanging process between the individual's mindset and the environment is a fundamental component of the mindsponge mechanism, understanding the studied problem's contexts is required (e.g., environmental, economic, and socio-cultural contexts).

\section{Step 2 (Identifying factors and their proxies)}

After determining the research problem and its context, the user needs to identify the factors of the problems and their proxies. During this process, the user should be greatly conscious of the feasibility of employing available data for choosing studied factors for the sake of cost-saving [1ㄷ].

\section{Step 3 (Constructing logical framework)}

The mindsponge mechanism should be interpreted as a framework to construct logical connections between factors of the investigated problem. Thus, after gathering enough factors to build a model, the user can create a logical network based on the reasoning principles of the mindsponge framework.

\section{Step 4 (Constructing and fitting model)}

Bayesian analysis programs, such as the bayesvl package, offer Bayesian inference calculation and 
support constructing a logical network before model fitting [21]. The user can be highly flexible in constructing a logical network due to the advantages of Bayesian inference.

\section{Step 5 (Interpreting and evaluating results)}

Finally, the simulated results are interpreted and evaluated. Suppose the simulated results are found to be ambiguous or illogical. In that case, the users should return to step 1 to identify whether the lack of consideration of mindsponge components induces ambiguity. Then, the procedure is repeated accordingly.

In general, the mindsponge mechanism can be considered the theoretical backbone to identify problems, construct the logical framework, and determine proxies for variables. At the same time, the logical construction and flexibility of Bayesian inference (e.g. bayesvl package) help construct and fit the model rationalized by the mindsponge mechanism.

\section{References}

1. Suzana, HH. (2012). The remarkable, yet not extraordinary, human brain as a scaled-up primate brain and its associated cost. Proceedings of the National Academy of Sciences, 109, 10661-10668. https://doi.org/10.1073/pnas.1201895109

2. Nguyen MH, Le TT, Nguyen HKT, Ho MT, Nguyen HTT, Vuong QH. (2021). Alice in Suicideland: Exploring the suicidal ideation mechanism through the sense of connectedness and help-seeking behaviors. International Journal of Environmental Research and Public Health, 18(7), 3681. https://doi.org/10.3390/ijerph18073681

3. Vuong QH. (2016). Global mindset as the integration of emerging socio-cultural values through mindsponge processes: A transition economy perspective. In J. Kuada (Ed.), Global Mindsets: Exploration and Perspectives (pp. 123-140). New York: Routledge

4. Vuong QH, Napier NK. (2015). Acculturation and global mindsponge: An emerging market perspective. International Journal of Intercultural Relations, 49, 354-367. https://doi.org/10.1016/j.ijintrel.2015.06.003

5. Vuong QH, La VP, Nguyen MH, Ho MT, Ho MT, Mantello P. (2020). Improving Bayesian statistics understanding in the age of Big Data with the bayesvl R package. Software Impacts, 4, 100016. https://doi.org/10.1016/j.simpa.2020.100016

6. Vuong QH, La VP, Nguyen MH, Ho MT, Tran T, Ho MT. (2020). Bayesian analysis for social data: A step-by-step protocol and interpretation. MethodsX, 7, 100924. https://doi.org/10.1016/j.mex.2020.100924

7. Vuong QH, Nguyen MH, Le TT. (2021). A Mindsponge-Based Investigation into the Psycho-Religious Mechanism Behind Suicide Attacks. Warsaw, Poland: De Gruyter / Sciendo. https://doi.org/10.2478/9788366675599 Online ISBN: 9788366675599

8. Vuong QH, Nguyen MH, Le TT. (2021). Home scholarly culture, book selection reason, and academic performance: Pathways to book reading interest among secondary school students. European Journal of Investigation in Health, Psychology and Education, 11(2), 468-495. https://doi.org/10.3390/ejihpe11020034

9. Vuong QH, Le TT, Nguyen QL, Nguyen QT, Nguyen MH. (2020). Escaping from air pollution: The psychological process of domestic migration intention among urban people. arXiv, 2108.00497. https://arxiv.org/abs/2108.00497

10. Phenikaa ISR. (2021). Đại diện Trung tâm ISR thuyết trình tại seminar do VIASM tổ chức. Retrieved from: https://isr.phenikaa-uni.edu.vn/vi/post/tin-tuc/tin-tuc/sub-tin-tuc/dai-dien-trung-tam-isr-thuyet-trinh-tai-seminar-doviasm-to-chuc

11. van Ravenzwaaij D, Cassey P, Brown SD. (2016). A simple introduction to Markov Chain Monte-Carlo sampling. Psychonomic Bulletin Review, 25(1), 143-154. https://doi.org/10.3758/s13423-016-1015-8

12. Dunson DB. (2001). Commentary: practical advantages of Bayesian analysis of epidemiologic data. American Journal of Epidemiology, 153(12), 1222-1226. https://doi.org/10.1093/aje/153.12.1222

13. Green PJ, Łatuszyński K, Pereyra M, Robert CP. (2015). Bayesian computation: a summary of the current state, and samples backwards and forwards. Statistics Computing, 25(4), 835-862. https://doi.org/10.1007/s11222-015-9574-5

14. Schaffer J. (2014). What not to multiply without necessity. Australasian Journal of Philosophy, 93(4), 644-664. https://doi.org/10.1080/00048402.2014.992447

15. Vuong QH. (2018). The (ir)rational consideration of the cost of science in transition economies. Nature Human Behaviour, 2, 5. https://doi.org/10.1038/s41562-017-0281-4

16. Halsey LG, Curran-Everett D, Vowler SL, Drummond GB. (2015). The fickle P value generates irreproducible results. Nature Methods, 12(3), 179-185. https://doi.org/10.1038/nmeth.3288

17. Vuong QH, Ho MT, La VP. (2019). 'Stargazing' and p-hacking behaviours in social sciences: some insights from a 
developing country. European Science Editing, 45(2), 54-55. http://europeanscienceediting.org.uk/articles/stargazingand-p-hacking-behaviours-in-social-sciences-some-insights-from-a-developing-country/

18. Kerr NL. (1998). HARKing: Hypothesizing after the results are known. Personality Social Psychology Review, 2(3), 196217. https://doi.org/10.1207/s15327957pspr0203_4

19. Baker M. (2016). 1,500 scientists lift the lid on reproducibility. Nature News, 533(7604), 452-454. Retrieved from: https://www.nature.com/news/1-500-scientists-lift-the-lid-on-reproducibility-1.19970

20. Open Science Collaboration. (2015). Estimating the reproducibility of psychological science. science, 349(6251), aac4716. https://doi.org/10.1126/science.aac4716

21. Nguyen MH. (2021). Bayesian inference: The enabler of Mindsponge philosophy. OSF Preprints. DOI: https://doi.org/10.31219/osf.io/n3jcx.

\section{Keywords}

Mindsponge mechanism; bayesvl;Bayesian analysis;psychology;cognitive sciences

Retrieved from https://encyclopedia.pub/13852 